\title{
Characterization of Tetragonal-Monoclinic, Ferroelastic Transformation and Domain Boundaries in Zirconia-Alloyed Yttrium Tantalate
}

\author{
Mor Baram ${ }^{1,2}$, Samuel Shian ${ }^{2}$, Mary Gurak ${ }^{2}$, Jing Feng ${ }^{2}$, Pankaj Sarin ${ }^{3}$, Waltraud M. Kriven ${ }^{4}$ and David \\ R. Clarke ${ }^{2}$ \\ 1. Department of Materials Science and Engineering, McMaster University, Hamilton, Ontario, Canada. \\ 2. School of Engineering and Applied Sciences, Harvard University, Cambridge, MA, USA. \\ 3. School of Materials Science and Engineering, Oklahoma State University, Tulsa, OK, USA. \\ 4. Department of Materials Science and Engineering, University of Illinois at Urbana Champaign, \\ Urbana, IL, USA.
}

There is a continuous search for an alternative oxide material to the yttria-stabilized zirconia (7YSZ), which currently serves as the thermal barrier coating materials for turbine engines [1]. The new material should have low thermal conductivity, high temperature stability, and ferroelastic toughening as exhibited by the 7YSZ. Therefore in this study, the Y-Ta-Zr-O system was investigated. The zirconia-rich part of this ternary phase diagram is quite studied, however less is known of the compositions in the vicinity of the $\mathrm{YTaO}_{4}$ phase. The goal of this study is to characterize the microstructure evolution that accompanied the tetragonal-monoclinic transformation in $\mathrm{YTaO}_{4}$ alloyed with 0 to 20 mole $\%$ of $\mathrm{ZrO}_{2}$.

Dense pellets were prepared from powders synthesized by the reverse co-precipitation. In this method, mixtures of zirconium oxynitrate, yttrium nitrate, and tantalum chloride precursor solutions were slowly added into ammonium hydroxide solution to initiate the formation of precipitates. After a series of washing to remove ammonium nitrate/chloride species, the gels were calcined at $700^{\circ} \mathrm{c}$ for 2 hours. The resulting powders where cold pressed at $100 \mathrm{MPa}$ and then sintered at $1500^{\circ} \mathrm{C}$ for 5 hours. From the pellets, transmission electron microscope (TEM) and atom probe tomography (APT) samples were prepared using a focused ion beam scanning electron microscope (FIB-SEM). The domains structure and orientation were analyzed using selected area electron diffraction (SAED) and high resolution TEM (HRTEM).

Examining the samples using electron microscopy revealed a twins-like domain structure in the monoclinic phase as can be seen in Figure 1. This form of domain structure is similar to the twinning effect that exists in other materials that exhibit ferroelastic toughening [2,3]. However, they differ from typical twins since the domains are related through rotation and not through mirror symmetry operation. These domains are sometimes has been referred to as type III mechanical twins [2]. In addition, the effect of the $\mathrm{ZrO}_{2}$ on the transformation temperature and microstructure was studied and discussed, and it was found that zirconia alloying decreases the transformation temperature.

In this work, the study of domain boundaries in monoclinic zirconia alloyed yttrium tantalate was conducted. We believe that this is the first evidence of such domains structure in this system. The transformation was found to be reversible and exhibited characteristics of ferroelastic, which is a critical feature for toughening at high temperature. The domains structure can support such conclusion. The zirconia alloying effect on transformation was also studied and correlated with the microstructure. The results are encouraging towards the future consideration of these phases as a substitute to the YSZ as thermal barrier coating. 


\section{References:}

[1]DR Clarke et al, Materials Research Bulletin 37 (2012) p. 891

[2]S Tsunekawa et al, Physica Status Solidi (a) 50 (1978) p. 695

[3] L Jian et al, Journal of the American Ceramic Society 79 (1996) p. 1642

[4] This work was supported at Harvard University by a grant from ONR (N00014-012-1-0993) and University of Illinois by the Air Force Office of scientific Research (FA9550-06-1-0386).
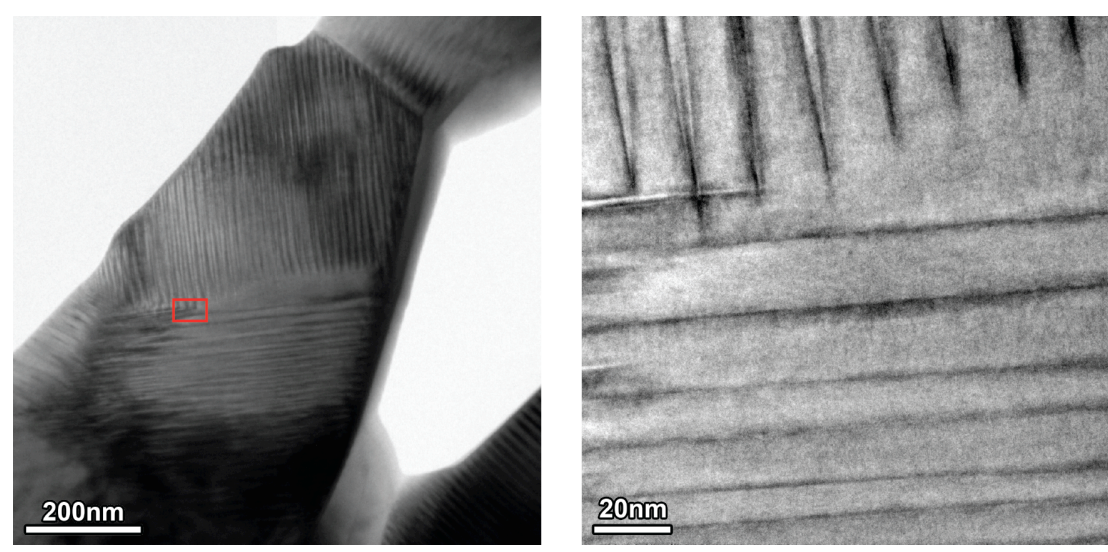

Figure 1. Low magnification (left) and high magnification (right) TEM micrograph presenting the domain structures observed in zirconia alloyed $\mathrm{YTaO}_{4}$. The small box on the left presents the area from which the high magnification micrograph was taken. 